\title{
Obesity-driven endometrial cancer: is weight loss the answer?
}

\author{
ML MacKintosh, ${ }^{a}$ EJ Crosbie ${ }^{b}$ \\ ${ }^{a}$ Department of Obstetrics \& Gynaecology, St Mary's Hospital, Manchester, UK ${ }^{b}$ Institute of Cancer Sciences, University of Manchester, \\ Manchester, UK \\ Correspondence: Dr E Crosbie, Institute of Cancer Sciences, University of Manchester, Fifth Floor Research, St Mary's Hospital, Oxford Road, \\ Manchester, M13 9WL, UK. Email emma.crosbie@manchester.ac.uk
}

Accepted 31 October 2012.

Please cite this paper as: MacKintosh M, Crosbie E. Obesity-driven endometrial cancer: is weight loss the answer?. BJOG 2013;120:791-794.

Endometrial cancer is the fourth most common cancer affecting British women, behind breast, lung and colon cancer. Over the past 20 years, the incidence of endometrial cancer has risen by $40 \%$, and deaths by $20 \%$, despite improved overall survival rates. Currently more than 1900 British women die from endometrial cancer each year, compared with fewer than 1500 at the turn of the century. ${ }^{1}$ The aging population, uterinesparing treatments for menstrual dysfunction and tamoxifen treatment for breast cancer have all contributed to this rise, but escalating obesity rates appear to be the major culprit.

Endometrial cancer ranks highest amongst all cancers in its association with obesity. Every $5 \mathrm{~kg} / \mathrm{m}^{2}$ increase in body mass index (BMI) confers a 1.6-fold increased risk of endometrial cancer. At a BMI of 42, a woman has an almost ten-fold increased risk of endometrial cancer than women of normal weight. ${ }^{2}$ Rates of obesity in England and Wales have trebled over the last two decades, and they are still on the increase. Currently, two-thirds of British women are overweight and nearly one-quarter obese. In Europe, excess weight has been estimated to account for $60 \%$ of all new endometrial cancer cases per year. ${ }^{3}$ Indeed, in the ASTEC trial, a European study of more than 1400 women with earlystage endometrial cancer, $80 \%$ of women with type- 1 endometrial cancer were overweight $(\mathrm{BMI}>25)$ and $50 \%$ were obese $(\mathrm{BMI}>30) .{ }^{4}$ Thus, although an average woman has a $3 \%$ lifetime risk of endometrial cancer, an obese woman has a risk of $9-10 \%$, and for morbidly obese women the risk may be even higher.

\section{How could obesity cause endometrial cancer?}

There are two types of endometrial cancer: endometrioid (type-1) cancer and non-endometrioid (type-2) cancer.
Obesity is thought to play a major aetiological role in the development of endometrioid tumours but not nonendometrioid tumours, which include several high-risk histotypes that develop via different pathways. Type-1 disease accounts for over $80 \%$ of cases, and even though it has a lower death rate than type- 2 disease, it still accounts for the majority of deaths. ${ }^{4}$ Obesity is a toxic state of hyperestrogenaemia, insulin resistance and inflammation (Figure 1). Obesity causes estrogenic excess because adrenal androgens are aromatised to estrogen by adipose tissue. ${ }^{5}$ In addition women who are obese have increased bioavailability of free estrogens because of low levels of sex hormone binding globulin (SHBG), a consequence of hyperinsulinaemia. Estrogens bind directly to endometrial cell DNA to increase transcription and interact with several growth factor signalling pathways, including the PI3K-Akt-mTOR and MAPK/ERK1,2 pathways, to promote proliferation. ${ }^{6}$ Excessive or 'unopposed' estrogenic stimulation of the endometrium, for example in postmenopausal or chronic anovulatory states (e.g. polycystic ovary syndrome, PCOS), drives tumourigenesis. ${ }^{5}$ Progestogens, naturally released during the second half of the menstrual cycle in ovulating women, or delivered exogenously, 'protect' the endometrium from the tumourigenic effects of estrogen. ${ }^{7}$

Obesity may also increase endometrial cancer risk through hyperinsulinaemia and insulin resistance. ${ }^{8}$ Increased circulating levels of insulin lead to reduced blood levels of insulinlike growth factor (IGF) binding proteins, contributing to increased bioavailability of IGF-1. IGF-1 is a growth factor that drives cellular proliferation through the activation of the PI3K-Akt-mTOR pathway. Inhibitors of this pathway include adiponectin, an adipokine found in the serum at concentrations inversely related to insulin resistance. Low 


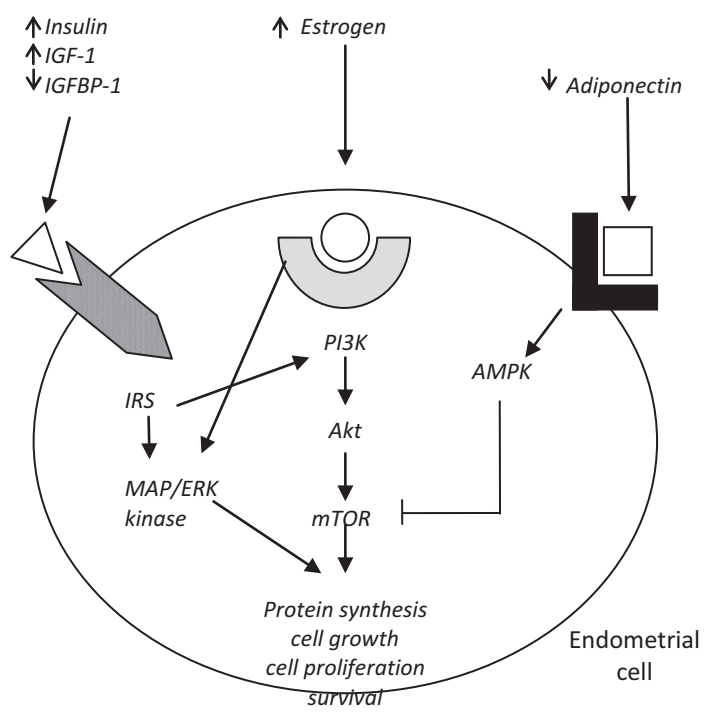

Figure 1. Mechanisms underpinning obesity-related endometrial carcinogenesis include the effects of hyperestrogenism, insulin resistance, altered serum adipokine concentrations and proinflammatory responses (not shown). Key: $A R$, adiponectin receptors; ER, estrogen receptor; IGF-1R, IGF-1 receptor; IR, insulin receptor.

adiponectin levels are a surrogate marker for insulin resistance, and correlate strongly with endometrial cancer risk, independent of BMI. Adiponectin inhibits the mammalian target of rapamycin (mTOR) pathway through the activation of AMP-activated protein kinase (AMPK). Obesity is a chronic inflammatory state associated with elevated systemic proinflammatory cytokines, promoters and hormones, including tumour necrosis factor alpha (TNF- $\alpha$ ), interleukin-6 (IL-6), C-reactive protein (CRP) and leptin. ${ }^{8}$ Proinflammatory markers have been implicated in tumourigenesis via their direct and indirect effects on cells of the innate and adaptive immune systems, as well as through disturbed tissue homeostasis and increased oxidative stress.

\section{The endometrial effects of obesity}

Despite the striking correlations observed between obesity and risk of endometrial cancer, few studies have investigated the effects of obesity on the endometrium. Hysterectomy specimens from women of normal weight (BMI 20-25), and from women who are overweight and obese, show that endometrial proliferation and the activation of key proproliferative signalling proteins (pAkt, pERK1,2) are correlated with BMI in premenopausal women. Compared with women of normal weight, women who are obese show increased endometrial proliferation, as evidenced by increased ki-67 expression, as well as increased phosphorylation of Akt (pAkt, 1.6-fold), ERK1,2 (pERK1,2 8.7-fold), despite having normal endometrial histology. 9 Thus the effects of obesity can be observed at a molecular level in apparently normal premenopausal endometrium. Endometrial cancer and its precursor lesion, atypical endometrial hyperplasia, usually present early following the onset of abnormal vaginal (typically postmenopausal) bleeding. However, clinically overt endometrial hyperplasia and neoplasia may be the tip of the iceberg. In a study of asymptomatic obese women, the prevalence of occult endometrial hyperplasia and cancer was 12 and 3\% in postmenopausal women, and 5.8 and $1 \%$ in premenopausal women, respectively $(n=193) .{ }^{10}$ The natural history of occult endometrial pathology is unknown.

\section{Can weight loss reduce endometrial cancer risk?}

Emerging evidence suggests that massive, sustained weight loss following bariatric surgery (gastric bypass or banding to reduce stomach capacity) reduces the risk of endometrial cancer. In a prospective Swedish study of more than 4000 matched patients who were morbidly obese undergoing bariatric surgery or medical weight loss management, a sustained weight loss of $20 \mathrm{~kg}$ for 10 years or more in the bariatric surgery group was associated with a $38 \%$ reduction in cancer incidence amongst women, but not men. ${ }^{11} \mathrm{~A}$ retrospective study of 4977 women who were morbidly obese from Virginia, USA, found a 38\% decrease in cancer incidence in the bariatric surgery group compared with controls who were obese, with pronounced reductions in the incidence of endometrial and postmenopausal breast cancer. ${ }^{12}$ In another retrospective study, a $24 \%$ reduction in incident cancers and $46 \%$ reduction in cancer mortality were seen amongst 6596 women undergoing bariatric surgery, compared with 9442 controls who were obese, over a 12.5year follow-up period in Utah, USA. ${ }^{13}$ The lack of an association seen for men may reflect the small numbers of men undergoing bariatric surgery (just 14\% of the surgical population in the latter study) and the increased effect of weight loss on the incidence of sex steroid-related tumours, such as breast and endometrial cancer, which predominantly and exclusively affect women, respectively. Interestingly, the most impressive reduction in cancer-specific incidence in the latter study was seen for endometrial cancer, where bariatric surgery resulted in a seven-fold reduction in incident cancer risk (14 cases in 6596 bariatric surgery patients versus 98 cases in 9442 controls who were obese, HR 0.22, $P<0.0001) .{ }^{13}$ Given that amongst all obesity-related malignancies the link with elevated BMI is strongest for endometrial tumours, this finding is perhaps unsurprising.

Taken together, these observations are exciting and provide proof of principle that endometrial cancer is preventable. Preventing endometrial cancer by weight loss is an attractive strategy as weight loss would also improve cardiovascular fitness, reduce/treat type-2 diabetes and 
reduce the risk of other obesity-related cancers, e.g. breast and colon cancer. ${ }^{14}$ Bariatric surgery is an effective means of achieving weight loss, but it is not feasible at a population level for reducing cancer risk. Alternative weight loss strategies have not demonstrated convincing efficacy or sustainability. Tackling obesity through diet and exercise is extremely challenging, and is likely to involve complex societal and political initiatives. Meanwhile, the obesity epidemic continues unabated throughout the UK, as well as much of Europe and North America.

The mechanism by which weight loss reduces endometrial cancer risk is complex. Bariatric surgery results in an almost instantaneous improvement in insulin resistance, with measurable effects on metabolism, serum glucose and insulin levels observed far in advance of any appreciable weight loss. Up to $30 \%$ of type- 2 diabetic patients are discharged home 2-3 days after bariatric surgery with no antiglycaemic medication and normal blood glucose levels. ${ }^{14}$ Patients may expect to lose $10-15 \%$ of their excess body weight by 6 weeks after surgery, with continued weight loss, albeit at a slower rate, up to about 1 year post-surgery. In general, patients settle at a BMI of 25-30; resolution to a normal weight BMI may never be achieved. The loss of adiposity reduces the aromatisation of adrenal androgens to estrogen, leading to a drop in serum estrogen levels. This is accompanied by higher plasma SHBG concentrations, leading to less bioavailable estrogen in the blood, although the nadir may not be reached until 12 months or longer after surgery. The resolution of chronic inflammatory change may also be slow given that proinflammatory markers are released primarily from white adipose tissue. The effects of surgically induced weight loss on metabolism, plasma sex steroid levels and inflammatory responses are clearly complicated, but in combination they provide a biologically plausible mechanism for the reduction in cancer incidence observed, particularly for estrogensensitive tumours. ${ }^{14}$

\section{Can weight loss 'treat' endometrial hyperplasia and neoplasia?}

Given the central role of obesity in its pathogenesis, and with compelling evidence that surgically induced weight loss 'prevents' endometrial cancer, an interesting question arises: can weight loss also 'treat' established endometrial hyperplasia and cancer? The study by Argenta et al., published in the current issue of BJOG, set out to answer this question. ${ }^{15}$ From 45 paired endometrial samples taken before surgery and at 1 year following surgically induced weight loss in asymptomatic women who were morbidly obese, the authors identified a baseline prevalence of endometrial pathology of $6.8 \%$ in this largely premenopausal group. There were no cases of occult cancer or atypical endometrial hyperplasia, but three cases of simple hyperplasia and one of complex hyperplasia without atypia. Although not strictly premalignant, hyperplastic change of the endometrium may set the stage for genetic events that pose a steadily increasing risk of progression to cancer. ${ }^{16}$ All four cases of endometrial hyperplasia regressed during follow-up: two by 12 months (one simple and one complex hyperplasia), and the other two (both simple hyperplasia) by 18 months, following surgery. In two cases, regression may have been aided by 'treatment' with oral contraceptives. A further case, negative for pathology at surgery, developed simple hyperplasia at 12 months. With such small numbers, a largely premenopausal cohort and the exclusion of women with recent abnormal vaginal bleeding, this study is clearly insufficiently powered to provide conclusive or convincing evidence for the role of bariatric surgery in the treatment of endometrial hyperplasia. However, it does introduce the idea that some hyperplastic abnormalities of the endometrium may be reversible through weight loss. It is conceivable that hyperplasia without atypia could regress following the withdrawal of stimulating factors (e.g. hyperestrogenaemia and hyperinsulinaemia), but it is more difficult to envisage the reversal of atypia or frankly malignant change through weight loss alone. Nonetheless, the bariatric surgery model provides the perfect opportunity to study the molecular changes that underpin endometrial cancer risk reduction. An improved understanding of these will facilitate the development of novel prevention strategies for endometrial cancer.

\section{Disclosure of interests}

The authors report no conflicts of interest.

\section{Contribution to authorship}

MLM and EJC. wrote this commentary together.

\section{Details of ethics approval}

No ethics approvals were required for this commentary.

\section{Funding}

MLM is a Clinical Research Fellow at Central Manchester University Hospitals NHS Foundation Trust; EJC is a Clinical Lecturer at the University of Manchester. No additional funding was required to complete this work.

\section{Acknowledgements}

We have no acknowledgments to make.

\section{References}

1 Cancer Research UK Cancer Statistics 2012. [http://info. cancerresearchuk.org/cancerstats/types/uterus/incidence/] Accessed 16 September 2012

2 Crosbie EJ, Zwahlen M, Kitchener HC, Egger M, Renehan AG. Body mass index, hormone replacement therapy and endometrial cancer risk: a meta-analysis. Cancer Epidemiol Biomarkers Prev 2010;19(12): 3119-30. 
3 Renehan AG, Soerjomataram I, Tyson $M$, Egger $M$, Zwahlen $M$, Coebergh JW, et al. Incident cancer burden attributable to excess body mass index in 30 European countries. Int I Cancer 2010;126:692-702.

4 Crosbie EJ, Roberts C, Qian W, Swart AM, Kitchener HC, Renehan AG. Body mass index does not influence post treatment survival in early stage endometrial cancer: results from the MRC ASTEC trial. Eur J Cancer 2012;48:853-64.

5 Kaaks R, Lukanova A, Kurzer MS. Obesity, endogenous hormones, and endometrial cancer risk: a synthetic review. Cancer Epidemiol Biomark Prev 2002;11:1531-43.

6 Matias-Guiu X, Catasus L, Bussaglia E, Lagarda H, Garcia A, Pons C, et al. Molecular pathology of endometrial hyperplasia and carcinoma. Human Pathol 2001;32:569-77.

7 Furness S, Roberts H, Marjoribanks J, Lethaby A. Hormone therapy in 'postmenopausal women and risk of endometrial hyperplasia. Cochrane Database Syst Rev2012;15:CD000402.

8 Khandekar MJ, Cohen P, Spiegelman BM. Molecular mechanisms of cancer development in obesity. Nature Rev Cancer 2011;11:886-95.

9 Villavicencio A, Aguilar G, Argüello G, Dünner C, Gabler F, Soto E, et al. The effect of overweight and obesity on proliferation and activation of AKT and ERK in human endometrial. Gynecol Oncol 2010;117:96-102.

10 Viola AS, Gouveia D, Andrade L, Aldrighi JM, Viola CF, Bahamondes L. Prevalence of endometrial cancer and hyperplasia in non-symptomatic overweight and obese women. Aust N Z J Obstet Gynaecol 2008:48:207-13.
11 Sjostrom L, Gummesson A, Sjöström CD, Narbro K, Peltonen M, Wedel $\mathrm{H}$, et al. Swedish Obese Subjects Study. Effects of bariatric surgery on cancer incidence in obese patients in Sweden (Swedish Obese Subjects Study): a prospective, controlled intervention trial. Lancet Oncol. 2009;10:653-62.

12 McCawley GM, Ferriss JS, Geffel D, Northup CJ, Modesitt SC. Cancer in obese women: potential protective impact of bariatric surgery. J Am Coll Surg 2009;208:1093-8.

13 Adams TD, Stroup AM, Gress RE, Adams KF, Calle EE, Smith SC, et al. Cancer incidence and mortality after gastric bypass surgery. Obesity 2009; 17:796-802.

14 Ashrafian H, Ahmed K, Rowland SP, Patel VM, Gooderham NJ, Holmes $E$, et al. Metabolic surgery and cancer: protective effects of bariatric procedures. Cancer 2011;117:1788-99.

15 Argenta PA, Kassing M, Truskinovsky AM, Svendsen CA. Bariatric surgery and endometrial pathology in asymptomatic morbidly obese women: a prospective, pilot study. BJOG 2013;120:795-800.

16 Popat VC, Vora DN, Gadhvi NS, Bhanvadia VM, Madan YG. Comparison of endometrial intraepithelial neoplasia with WHO endometrial hyperplasia classification system. A compariative study of 150 cases. Histopathology 2010;57:646-8. 